\title{
RIPK3 mediates pathogenesis of experimental ventilator-induced lung injury
}

\author{
Ilias I. Siempos, ${ }^{1,2}$ Kevin C. Ma, ${ }^{1}$ Mitsuru Imamura, ${ }^{1}$ Rebecca M. Baron, ${ }^{3}$ Laura E. Fredenburgh, ${ }^{3}$ \\ Jin-Won Huh, ${ }^{4}$ Jong-Seok Moon, ${ }^{1}$ Eli J. Finkelsztein, ${ }^{1}$ Daniel S. Jones, ${ }^{1}$ Michael Torres Lizardi, ${ }^{5}$ \\ Edward J. Schenck, ${ }^{1}$ Stefan W. Ryter, ${ }^{1}$ Kiichi Nakahira, ${ }^{1}$ and Augustine M.K. Choi ${ }^{5}$ \\ 'Department of Medicine, Division of Pulmonary and Critical Care Medicine, New York-Presbyterian Hospital/Weill \\ Cornell Medical Center, Weill Cornell Medicine (WCM), New York, New York, USA. ${ }^{2}$ First Department of Critical Care \\ Medicine and Pulmonary Services, Evangelismos Hospital, University of Athens Medical School, Athens, Greece. \\ ${ }^{3}$ Division of Pulmonary and Critical Medicine, Brigham and Women's Hospital (BWH), Harvard Medical School, Boston, \\ Massachusetts, USA. ${ }^{4}$ Department of Pulmonary and Critical Care Medicine, Asan Medical Center, University of Ulsan \\ College of Medicine, Seoul, Korea. ${ }^{5}$ Department of Medicine, New York-Presbyterian Hospital/Weill Cornell Medical \\ Center, WCM, New York, New York, USA.
}

In patients requiring ventilator support, mechanical ventilation (MV) may induce acute lung injury (ventilator-induced lung injury [VILI]). VILI is associated with substantial morbidity and mortality in mechanically ventilated patients with and without acute respiratory distress syndrome. At the cellular level, VILI induces necrotic cell death. However, the contribution of necroptosis, a programmed form of necrotic cell death regulated by receptor-interacting protein-3 kinase (RIPK3) and mixed-lineage kinase domain-like pseudokinase (MLKL), to the development of VILI remains unexplored. Here, we show that plasma levels of RIPK3, but not MLKL, were higher in patients with MV (i.e., those prone to VILI) than in patients without MV (i.e., those less likely to have VILI) in two large intensive care unit cohorts. In mice, RIPK3 deficiency, but not MLKL deficiency, ameliorated VILI. In both humans and mice, VILI was associated with impaired fatty acid oxidation (FAO), but in mice this association was not observed under conditions of RIPK3 deficiency. These findings suggest that FAO-dependent RIPK3 mediates pathogenesis of acute lung injury.

Conflict of interest: The authors have declared that no conflict of interest exists.

Submitted: August 28, 2017

Accepted: April 4, 2018

Published: May 3, 2018

Reference information: JCI Insight. 2018;3(9):e97102. https:// doi.org/10.1172/jici.nsight.97102

\section{Introduction}

The clinical importance of ventilator-induced lung injury (VILI) was established when a randomized controlled trial reported that mechanical ventilation (MV) with high tidal volume (i.e., injurious MV) was associated with a $9 \%$ absolute increase in mortality of patients with acute respiratory distress syndrome (ARDS) $(1,2)$. Evidence later accumulated that VILI can take place even in patients without previously damaged lungs (3-5). At the cellular level, VILI induces necrotic cell death $(6,7)$. However, the contribution of necroptosis, a programmed form of necrotic cell death that is regulated by receptor-interacting protein-3 kinase (RIPK3) and mixed-lineage kinase domain-like pseudokinase (MLKL) $(8,9)$, in the development of VILI has not been defined. Although the necroptosis-related molecules (such as RIPK3 and MLKL) have been studied in several animal models of disease, conclusive evidence to support their role in human disease, including VILI, is lacking (9). Here, we hypothesized that necroptosis-related molecules contribute to the pathogenesis of VILI and that there is an association among these molecules, VILI, and impaired fatty acid oxidation (FAO). To test this hypothesis, we measured the plasma levels of RIPK3 and MLKL in two cohorts of patients hospitalized in the intensive care unit (ICU). In addition, we explored the roles of necroptosis-related molecules and impaired FAO in mouse VILI.

\section{Results}

To explore the involvement of necroptosis-related molecules in human VILI, plasma was collected from 155 individuals enrolled in the WCM Biobank and Registry of Critically Ill Patients, an ongoing prospective cohort of patients hospitalized in a medical ICU (10). Information on the characteristics of enrolled 
Table 1. Characteristics of patients in the two cohorts

\begin{tabular}{|c|c|c|}
\hline & WCM & BWH \\
\hline Number of patients, $n$ & 155 & 199 \\
\hline Female, $n(\%)$ & $72(47)$ & $85(43)$ \\
\hline European descent, $n$ (\%) & $96(62)$ & $150(75)$ \\
\hline Malignancy, n (\%) & $68(44)$ & $82(41)$ \\
\hline Mechanical ventilation, $n(\%)^{\mathrm{A}}$ & $51(33)$ & $67(34)$ \\
\hline ARDS, $n(\%)$ & $26(17)$ & $24(12)$ \\
\hline In-hospital mortality, n (\%) & $31(20)$ & $35(18)$ \\
\hline
\end{tabular}

${ }^{\text {AWithin }} 24$ hours after admission in the ICU. WCM, Weill Cornell Medicine; BWH, Brigham and Women's Hospital; IQR, interquartile range; ARDS, acute respiratory distress syndrome.

individuals is displayed in Table 1. Fifty-one (33\%) of the enrolled patients were undergoing MV at the time of blood draw. RIPK3 levels in plasma were higher in patients with MV (i.e., those prone to VILI) compared with patients without MV (i.e., those less likely to have VILI) (Figure 1A). In contrast, plasma receptor-interacting protein-1 kinase (RIPK1) levels were not different between patients with MV versus those without MV (Figure 1B), and there was no correlation between plasma RIPK3 and RIPK1 levels (Figure 1C). This was also the case for plasma MLKL levels. Plasma MLKL levels were not different between patients with MV versus those without MV (Figure 1D), and there was no correlation between plasma RIPK3 and MLKL levels (Figure 1E). Plasma RIPK3 levels were also measured in 199 individuals enrolled in the BWH Registry of Critical Illness (Boston, Massachusetts, USA), an ongoing prospective cohort of patients hospitalized in a medical ICU $(11,12)$. One hundred and twenty-eight $(64 \%)$ of the enrolled patients had sepsis and 34\% were undergoing MV at the time of blood draw (Table 1). Similar to the WCM cohort, plasma RIPK3 levels of patients enrolled in the BWH cohort were higher in subjects with MV versus those without MV (Figure 2A). VILI is more likely to occur and has more serious consequences in patients with ARDS (2). We found that plasma RIPK3 levels were higher in subjects with ARDS versus those without ARDS (Figure 2B). Because RIPK3 may be involved in sepsis and therefore presence of sepsis may alter plasma levels of RIPK3 $(13,14)$, we focused on the subgroup of patients who were diagnosed with sepsis, and we found the same result; plasma RIPK3 levels were higher in subjects with MV versus those without MV (Figure 2C). Consistently, a multivariable regression analysis demonstrated that MV was a statistically significant independent predictor of elevated RIPK3 levels in plasma of critically ill patients after adjustment for potential confounders, such as sepsis (Supplemental Table 1; supplemental material available online with this article; https://doi.org/10.1172/jci.insight.97102DS1). Taken together, human studies suggest that the necroptosis-related molecule RIPK3, but not RIPK1 or MLKL, is elevated in plasma of critically ill patients, who undergo MV and are therefore prone to VILI.

To explore the involvement of necroptosis-related molecules in mouse VILI, we defined the responses of mice deficient in these molecules (namely, RIPK3, RIPK1, and MLKL) after injurious MV. Under baseline conditions, there was no difference between Ripk $^{-1-}$ and WT mice in terms of body weight and total protein concentration in the bronchoalveolar lavage fluid (BALF) (Supplemental Figure 1, A and B). Injurious MV increased RIPK3 expression in the lung, as assessed by immunohistochemistry (Supplemental Figure 2). In accordance with previous studies using WT animals $(11,15)$, ventilation with high tidal volume (i.e., injurious MV), as opposed to ventilation with low tidal volume, led to VILI manifested by impaired lung mechanics, increased alveolar capillary permeability, and inflammation. These responses to injurious MV were attenu-

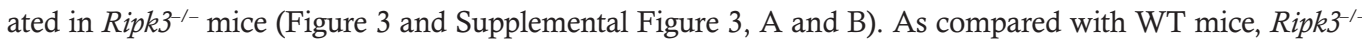
mice sustained a lower increase in lung parenchymal elastance (an indication of less impaired lung mechanics) (Figure 3A), a higher inspiratory capacity (a surrogate of less lung edema formation) (Figure 3B), a lower increase in tissue damping (another indication of less impaired lung mechanics) (Figure 3C), a lower total protein concentration in BALF (a surrogate of less impaired alveolar capillary permeability) (Figure 3D), and decreased markers of inflammation, such as IL-6 in BALF (Supplemental Figure 3, A and B). In addition, 
A

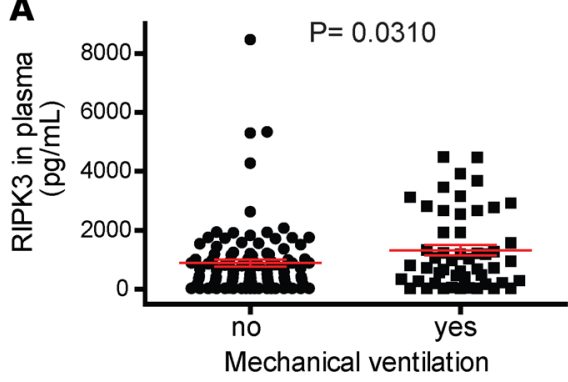

D

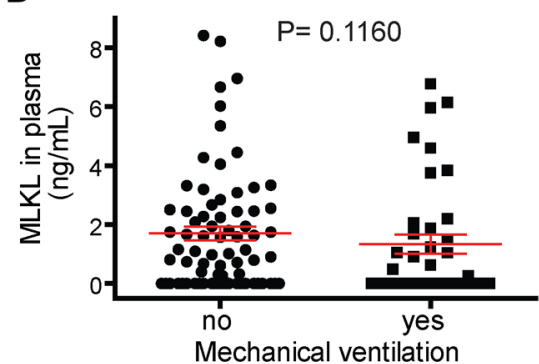

B

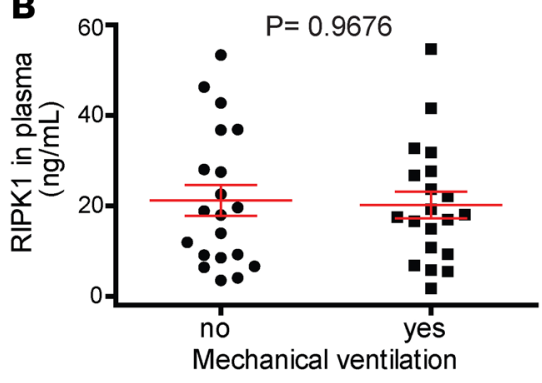

E

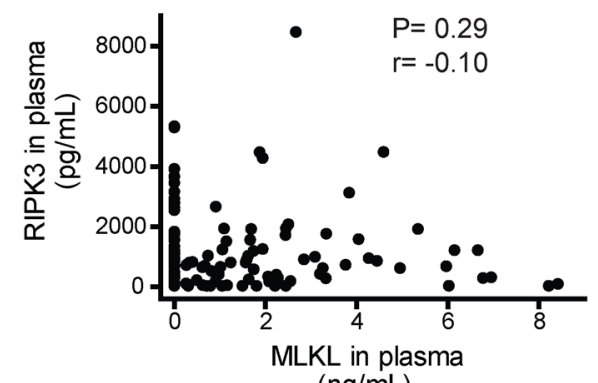

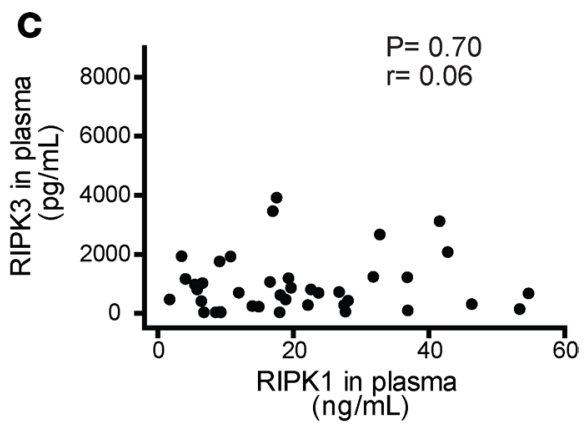

$(\mathrm{ng} / \mathrm{mL})$

Figure 1. Elevation of plasma RIPK3, but not RIPK1 or MLKL, levels in mechanically ventilated patients in the intensive care unit. (A) RIPK3 levels were plotted for patients with versus without mechanical ventilation (MV) in the Weill Cornell Medicine (WCM) cohort $(n=155)$. (B and C) For the WCM cohort, plasma RIPK1 levels were (B) plotted for patients with versus without MV and (C) correlated with plasma RIPK3 levels $(n=40)$. (D and E) For the WCM cohort, plasma MLKL levels were (D) plotted for patients with versus without MV and (E) correlated with plasma RIPK3 levels $(n=111)$. Red bars represent mean \pm SEM. Statistical significance was calculated using Mann-Whitney $U(\mathbf{A}, \mathbf{B}$, and $\mathbf{D})$ and Pearson's correlation $(\mathbf{C}$ and $\mathbf{E})$ tests.

lungs of Ripk3 ${ }^{-1-}$ mice may be resistant to necrosis associated with injurious MV, as evidenced by reductions in the release of lactate dehydrogenase (LDH) (Figure 3E) and chromatin protein high-mobility group B1 (HMGB1), a marker of necrosis (Figure 3F), in BALF compared with WT mice (16-18).

Because RIPK1 has been shown to form a complex with RIPK3, the necrosome, which has a pivotal role in necroptosis (19), we investigated the possible involvement of RIPK1 in the pathogenesis of VILI.

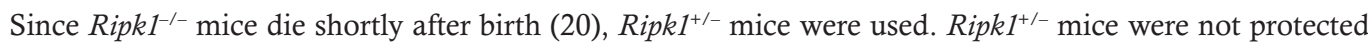
against injurious MV compared with $R i p k 1^{+/+}$mice (Supplemental Figure 4, A-E). This conclusion was further supported by the failure of necrostatin-1, a RIPK1 inhibitor (21), to confer protection against VILI (Supplemental Figure 5, A-E).

Because MLKL is considered a key component of necroptosis(22), we then tested whether MLKL is involved in the pathogenesis of VILI. We found that compared with $M l k l^{+/+}$mice, $M l k l^{-1}$ mice undergoing injurious
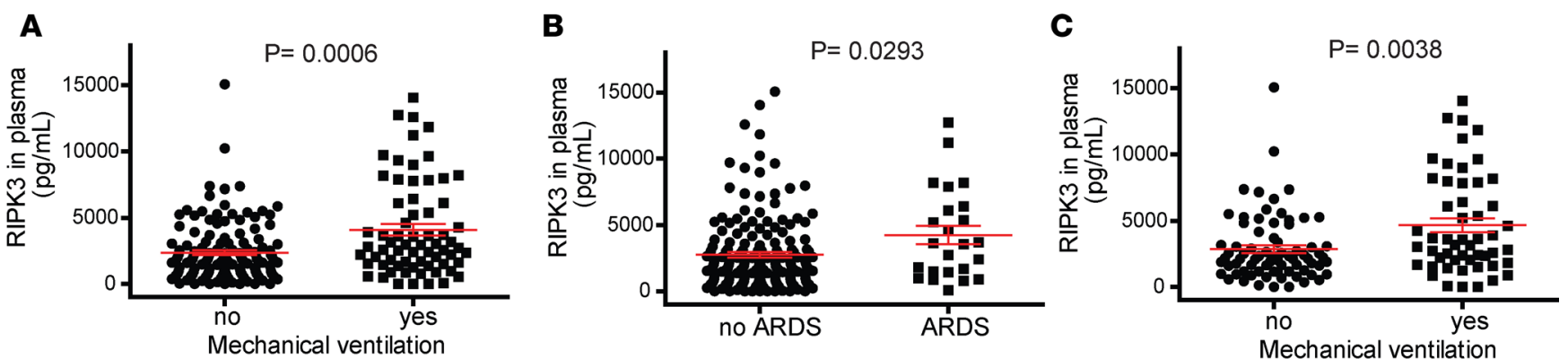

Figure 2. Elevation of plasma RIPK3 levels in mechanically ventilated patients in the intensive care unit (validation cohort). (A) RIPK3 levels were plotted for patients with versus without mechanical ventilation (MV) in the Brigham and Women's Hospital (BWH) cohort $(n=199)$. (B) For the BWH cohort, RIPK3 levels were compared in patients with versus without acute respiratory distress syndrome (ARDS) ( $n=199)$. (C) For the BWH cohort, RIPK3 levels were compared in patients with versus without MV among those who had sepsis $(n=128)$. Red bars represent mean \pm SEM. Mann-Whitney $U$ test was used for all comparisons. 
A

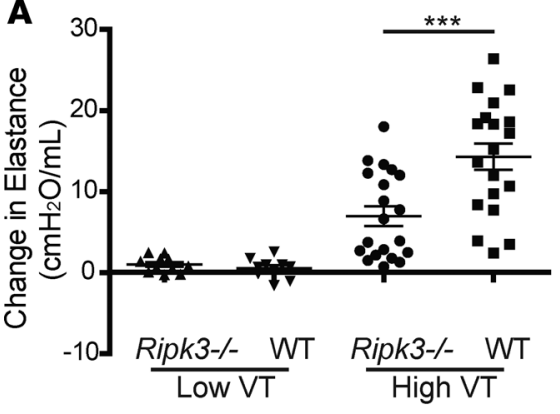

D

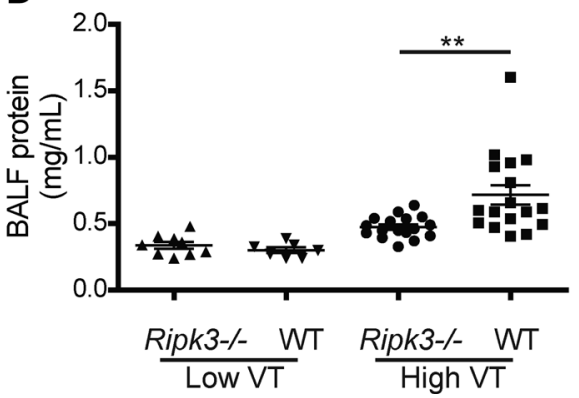

B

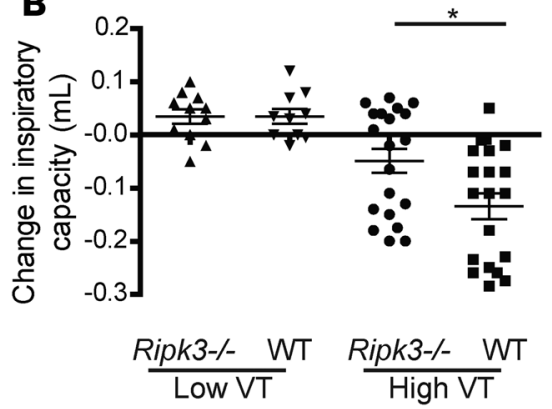

E

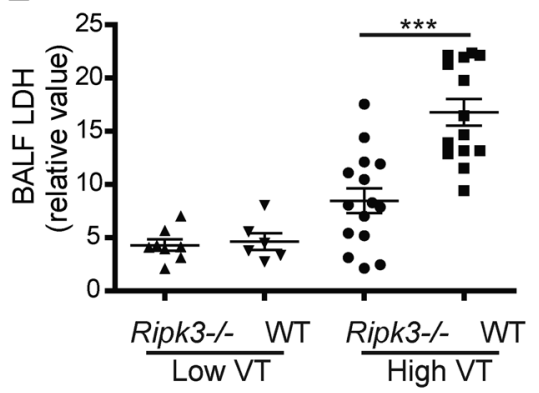

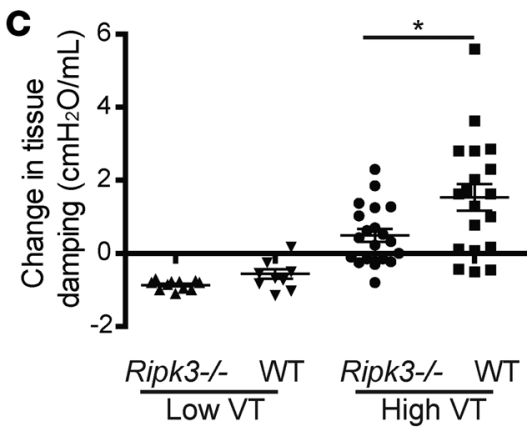

$\mathbf{F}$

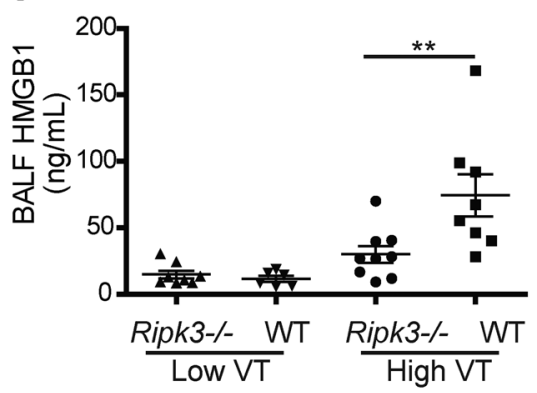

Figure 3. Role of RIPIK in ventilator-induced lung injury in mice. Ripk3-/- and WT mice underwent mechanical ventilation (MV) at low or high tidal volume (low VT or high VT). Changes in (A) lung elastance, (B) inspiratory capacity, and (C) tissue damping were recorded, and bronchoalveolar lavage fluid (BALF) was measured for (D) total protein concentration, (E) lactate dehydrogenase (LDH), and (F) chromatin protein high-mobility group B1 (HMGB1). Data are presented as mean \pm SEM $\left({ }^{*} P<0.05 ;{ }^{* *} P<0.01 ;{ }^{* *} P<0.001\right)$. ANOVA with Tukey post hoc correction was used for all comparisons.

MV were not protected against VILI (Figure 4 and Supplemental Figure 6). Taken together, these results indicate that RIPK3, but not RIPK1 or MLKL, may be indispensable in the pathogenesis of VILI in mice.

The above studies demonstrate that VILI is associated with RIPK3, and RIPK3 has been previously postulated to be linked to fatty acid metabolism (23). Thus, we next conducted studies to explore whether FAO is altered during VILI. In a human cohort of medical ICU patients (24), the ratio of free carnitine to palmitoylcarnitine plus oleylcarnitine $[\mathrm{C} 0 /(\mathrm{C} 16+\mathrm{C} 18)]$ was calculated in plasma. Elevation in this ratio is used as a newborn screening test for carnitine palmitoyl transferase (CPT1) deficiency in humans (25). We found that patients with ARDS had a higher $\mathrm{C} 0 /(\mathrm{C} 16+\mathrm{C} 18)$ ratio than those without ARDS (Figure $5 \mathrm{~A}$ ), and the trend persisted when the comparison involved patients with MV versus those without MV (Supplemental Figure 7). These findings suggest that CPT1 deficiency may be present in patients who are likely to have VILI. Interestingly, there was an association between the increase in the $\mathrm{C} 0 /(\mathrm{C} 16+\mathrm{C} 18)$ ratio (a marker of CPT1 deficiency) and the increase in plasma RIPK3 levels in critically ill patients (Figure 5B). Given that CPT1 mediates the rate-limiting step of $\beta$-oxidation of fatty acids through the control of their mitochondrial uptake $(26,27)$, the above results suggest that VILI may be associated with deregulated FAO in humans and that RIPK3 may play a role in this association. In an animal model of VILI, gas chromatography and liquid chromatography combined with detection by mass spectrometry were performed, and it was found that long-chain fatty acids, such as palmitic, palmitoleic, and linoleic acid, were elevated in lung tissue of mice exposed to injurious MV compared with controls (Figure 6A). In addition, free fatty acids were elevated in BALF of mice exposed to injurious MV as opposed to controls, but this increase was attenuated under conditions of RIPK3 deficiency (Figure 6B). Interestingly, there was a strong positive correlation between the free fatty acids in BALF and the severity of lung injury (as assessed by the BALF protein) (Figure 6C). The above results suggest that VILI may be associated with impaired FAO and, consequently, with an increase in fatty acids in mouse lung and that RIPK3 may play a role in this association.

To further support this conclusion, we tested the effect of the impairment of FAO, achieved by inhibition of CPT1, on the development of VILI. $C p t 1^{-/-}$mice are embryonically lethal, and, therefore, chemical inhibition of CPT1 was employed (26). Perhexiline, a CPT1 inhibitor that is clinically used as a second-choice antianginal treatment and is also tested for treatment of patients with congestive heart failure $(26,28,29)$, was administered 
A

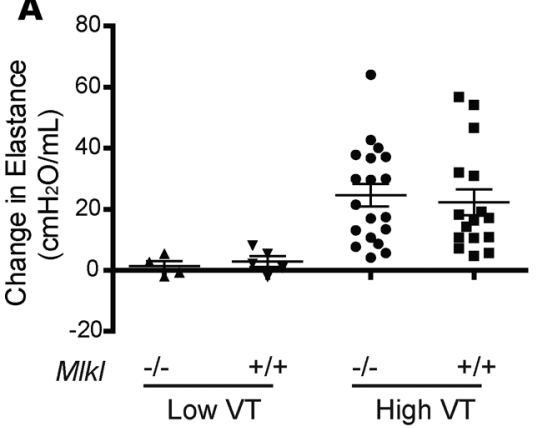

D

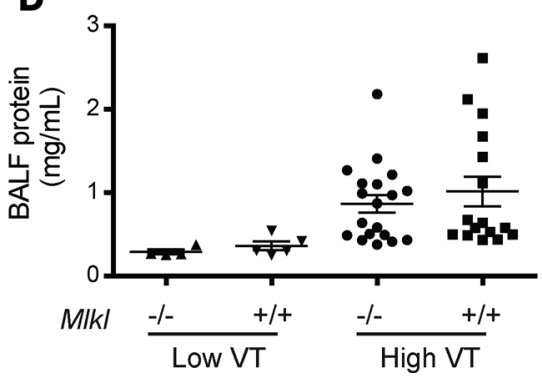

B

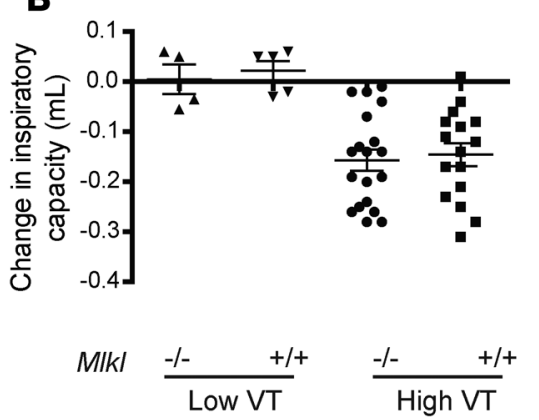

$\mathbf{E}$

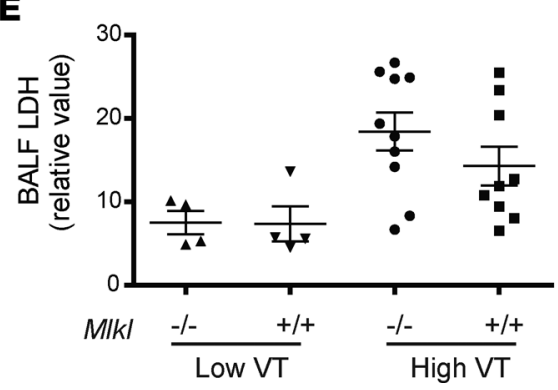

Figure 4. Role of MLKL in ventilator-induced lung injury in mice. $M I \mathrm{kl}^{-{ }^{--}}$and $\mathrm{MI} / \mathrm{kl}^{+/+}$mice underwent mechanical ventilation (MV) at low or high tidal volume (low VT or high VT). Changes in (A) lung elastance, (B) inspiratory capacity, and (C) tissue damping were recorded, and bronchoalveolar lavage fluid (BALF) was measured for (D) total protein concentration and (E) lactate dehydrogenase (LDH). Data are presented as mean \pm SEM. ANOVA with Tukey post hoc correction was used for all comparisons.

in WT mice undergoing MV with low or high tidal volume. In mice receiving low tidal volume, perhexiline did not exacerbate lung injury. However, in mice receiving high tidal volume, administration of perhexiline in a dose equivalent to that used in clinical practice for humans, as opposed to vehicle, led to worse VILI (Figure 7, A-C, and Supplemental Figure 8, A-C). We repeated the experiment by using another CPT1 inhibitor, etomoxir, which has been tested in clinical trials for the treatment of patients with congestive heart failure but was withdrawn due to its toxicity $(26,30)$. Similar to perhexiline, in mice receiving MV at high tidal volume, but not those at low tidal volume, administration of etomoxir was associated with worse VILI compared with vehicle (Supplemental Figure 9, A-F). These experiments suggest an association between VILI and impaired FAO in mice.

Finally, to determine whether RIPK3 played a role in the observed association between VILI and inhibition of FAO, Ripk $3^{--}$and WT mice were treated with perhexiline and were subsequently exposed to injurious MV. Ripk $3^{-1-}$ mice again sustained less severe VILI than WT mice (Figure 7, D-F, and Supplemental Figure 10, A-D). These studies suggest that the association between VILI and impaired FAO was not present under conditions of RIPK3 deficiency in mice.

\section{Discussion}

We demonstrate that the necroptosis-related molecule RIPK3, but not RIPK1 or MLKL, may be important in the development of experimental VILI and that impaired FAO may be involved. Evidence is accumulating that RIPK3, either acting as a signaling scaffold or as a kinase, may have a role in cellular processes other than necroptosis (31). Indeed, Ripk $3^{-/-}$but not $\mathrm{Mlkl}^{-1-}$ mice are protected in a variety of disease models $(14,16,32)$. Our study suggests that VILI could be included in the disease models for which RIPK3 and MLKL exhibit discrepant roles. Moreover, our study contributes to the accumulating evidence supporting a functional linkage between necroptosis-related molecules and fatty acid metabolism $(23,33)$.

Our study has several limitations. First, although we had data specifying that MV was initiated before the blood draw in $51(33 \%)$ patients in the WCM cohort and 67 (34\%) patients in the BWH cohort (as depicted in Table 1), we did not have sufficient data to determine for how many hours each patient was undergoing MV prior to the blood draw. Given that plasma RIPK3 levels for a given patient are not likely to be constant after initiation of MV (and presumably onset of VILI), the exact time from initiation of MV to blood draw for measurement of RIPK3 levels is an important variable that we could not address in our study. 

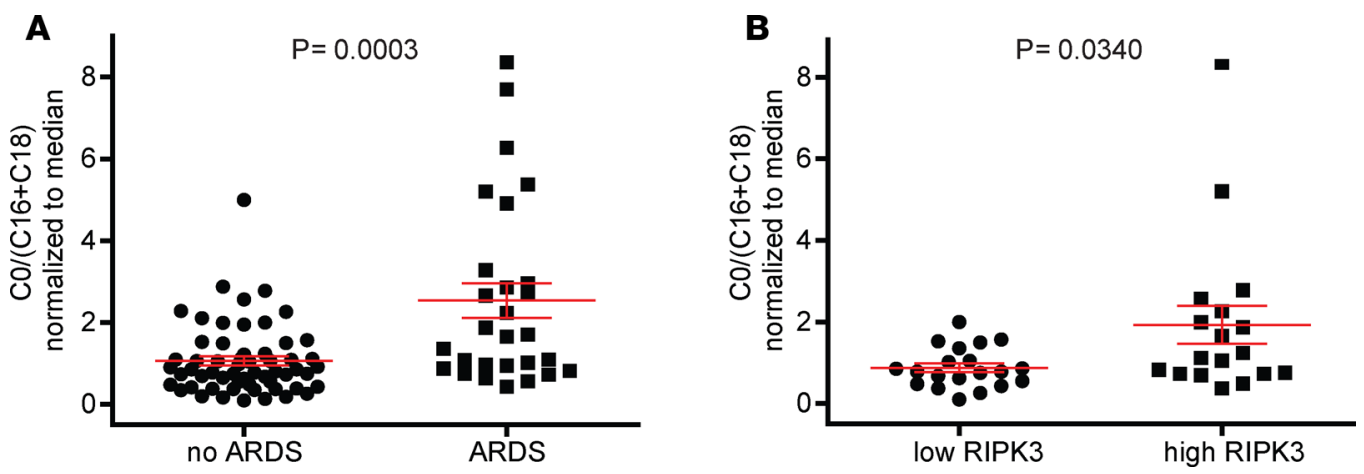

Figure 5. Defective fatty acid oxidation in critically ill patients. (A) Calculation of free carnitine to palmitoylcarnitine plus oleylcarnitine ratio [CO/(C16+C18), a screening test for deficiency of carnitine palmitoyl transferase (CPT1), which is a key enzyme in the fatty acid oxidation pathway] in plasma of patients with versus without acute respiratory distress syndrome (ARDS) $(n=86)$. (B) Critically ill patients, for whom both metabolomic data and plasma RIPK3 levels were available, were categorized into a "low RIPK3" group (i.e., those with plasma RIPK3 levels equal to or lower than median) and "high RIPK3" group (i.e., those with plasma RIPK3 levels higher than median) and were compared in terms of the ratio $\mathrm{CO} /(\mathrm{C} 16+\mathrm{C} 18)(n=38)$. Red bars represent mean \pm SEM. Statistical significance was calculated using Mann-Whitney $U$ test.

Second, patients enrolled in our first cohort (WCM) had a lower median and a narrower distribution of plasma RIPK3 levels than patients in the second cohort (BWH). Given that both cohorts had similar inclusion criteria, the above differences in plasma RIPK3 levels might be explained by differences in the duration of plasma storage. Indeed, enrollment in the WCM cohort (between 10/2014 and 07/2016) took place more recently and for a narrower period than enrollment in the BWH cohort (between 07/2008 and 09/2013). We should emphasize that this explanation is only hypothetical and that the reason for the differences in RIPK3 levels in the two cohorts is not clear. That said, the fact that our main finding (i.e., that plasma RIPK3 levels are higher in MV patients) persisted despite such differences between the cohorts apparently demonstrates the generalizability of our observations.

Third, given that there is no gold standard for diagnosis of VILI in the clinical setting (1), we used MV as its surrogate on the basis that MV is a definite risk factor for VILI (1). Unfortunately, we did not consistently collect extensive data on physiologic and ventilator parameters, such as driving pressure or tidal volume, which could permit identification of patients undergoing suboptimal MV (as indicated, e.g., by high driving pressure or high tidal volume). Therefore, we could not conduct an analysis of plasma RIPK3 levels
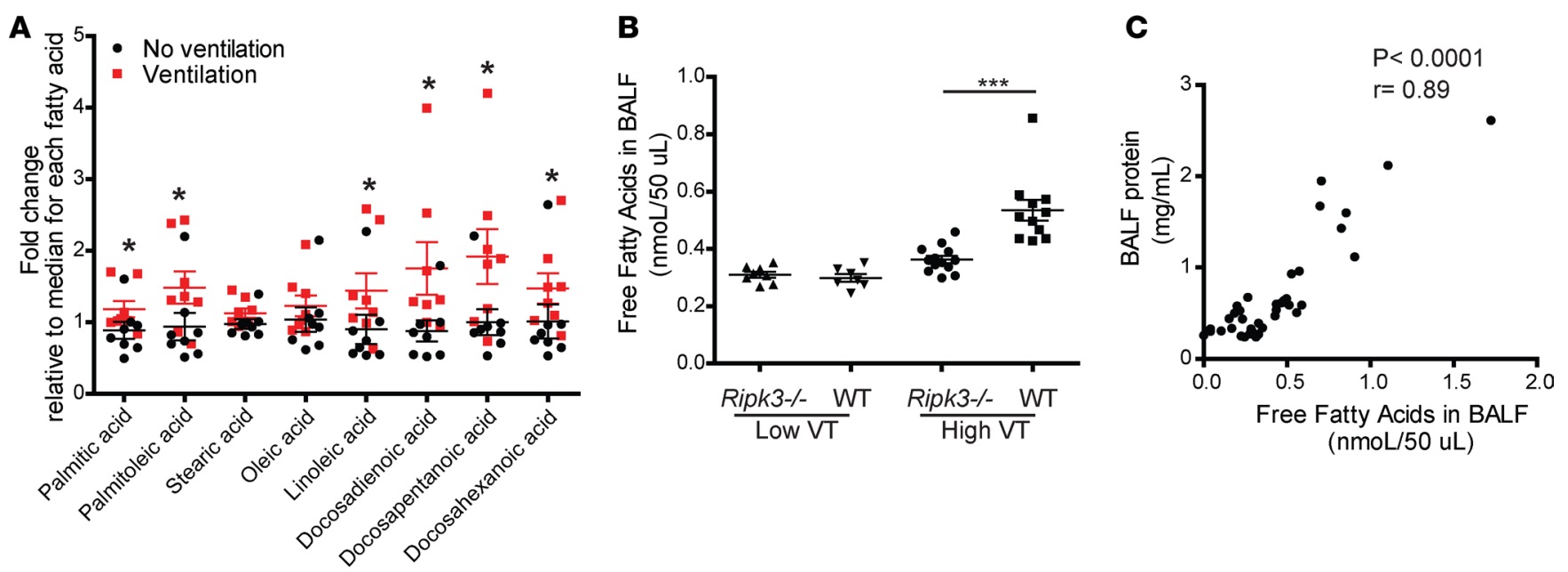

Figure 6. Defective fatty acid oxidation in ventilator-induced lung injury in mice. (A) Fatty acid quantification in lung tissue of WT mice with versus without mechanical ventilation (MV), determined by gas chromatography and liquid chromatography combined with detection by mass spectrometry. (B and C) After MV at low or high tidal volume (low VT or high VT), free fatty acids were (B) measured in bronchoalveolar lavage fluid (BALF) of Ripk $3^{-1-}$ and WT mice and (C) correlated with total protein concentration in BALF. Data are presented as mean \pm SEM $\left({ }^{*} P<0.05 ;{ }^{* * *} P<0.001\right)$. Statistical significance was calculated using Mann-Whitney $U$ (A), ANOVA with Tukey post hoc correction (B), and Pearson's correlation (C) tests. 
A
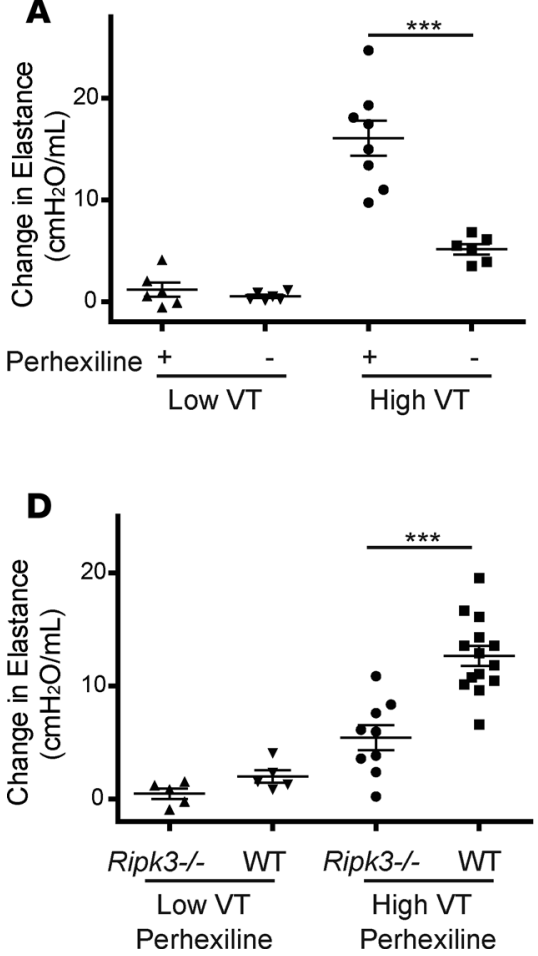

B

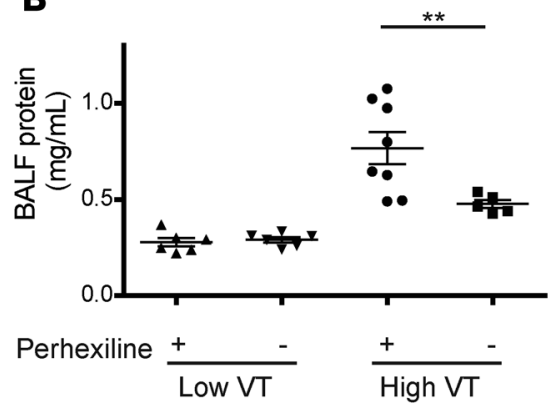

$\mathbf{E}$

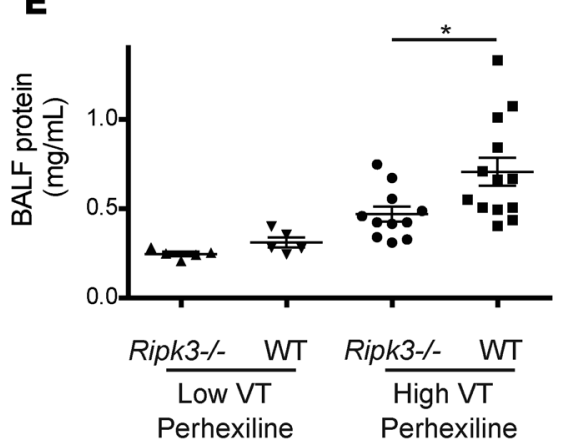

C

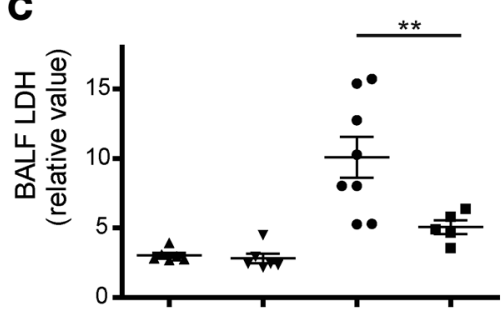

Perhexiline $\frac{+}{\text { Low VT }} \quad \frac{+}{\text { High VT }}$

$\mathbf{F}$

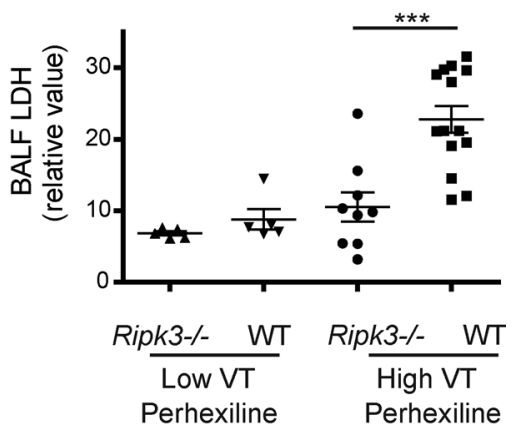

Figure 7. Administration of carnitine palmitoyl transferase inhibitor in mice and exposure to mechanical ventilation. (A-C) Changes in (A) lung elastance were recorded, and bronchoalveolar lavage fluid (BALF) was measured for (B) total protein concentration and (C) lactate dehydrogenase (LDH) in WT mice after treatment or not with a carnitine palmitoyl transferase (CPT1) inhibitor, perhexiline, and exposure to mechanical ventilation (MV) at low or high tidal volume (low VT or high VT). (D-F) Changes in (D) lung elastance were recorded, and BALF was measured for (E) total protein concentration and (F) LDH in Ripk $3^{-/-}$and WT mice after treatment with perhexiline and exposure to MV at low or high tidal volume. Data are presented as mean \pm SEM $\left({ }^{*} P<0.05 ;{ }^{* *} P<0.01 ;{ }^{* *} P<0.001\right)$. ANOVA with Tukey post hoc correction was used for all comparisons.

in this subgroup of patients who were very likely to have VILI. Such a subgroup analysis would further support the association between RIPK3 and human VILI. However, we conducted a sensitivity analysis of patients with ARDS and found that plasma RIPK3 levels were higher in patients with ARDS versus those without ARDS (Figure 2B). ARDS is an even stronger risk factor for VILI than MV alone, and it has been extensively used in the literature (both clinical and animal) as a surrogate of VILI $(1,2,6)$. Taken together, we should emphasize that, given the lack of a gold standard for diagnosis of human VILI (1), one cannot be sure regarding the association between RIPK3 and human VILI in specific (as opposed to acute lung injury in general). Rather, the safest interpretation of our clinical studies is that, given that MV is a risk factor of lung injury and that plasma RIPK3 levels were higher in patients with MV than in patients without MV, elevated RIPK3 levels may be a marker of acute lung injury, including, but not limited to VILI.

In conclusion, our analysis of clinical data from two large and well-phenotyped cohorts provide evidence that RIPK3 levels are elevated in critically ill patients undergoing MV (and who are therefore prone to VILI) compared with those who are not undergoing MV (and who are therefore less likely to have VILI). Consistently, our animal experiments highlight the contribution of RIPK3, as well as the potential involvement of impaired FAO, to the pathogenesis of VILI. Taken together, our clinical and animal data may justify a prospective clinical study to examine whether plasma RIPK3 levels can predict worse clinical outcomes of critically ill patients undergoing MV.

\section{Methods}

Human cohorts. Study subjects were adult ( $\geq 18$-year-old) patients enrolled in two ongoing prospective registries of critically ill patients hospitalized in a medical ICU in the New York-Presbyterian Hospital/WCM (enrollment started on 10/2014) and in the BWH (enrollment started on 07/2008). Patients were excluded if they were unwilling to provide biological samples for research, mentally handicapped, unable to provide informed consent, admitted merely to receive comfort care, unwilling to be transfused, or if they had a 
hemoglobin level of $<7 \mathrm{~g} / \mathrm{dl}$ upon admission (10-12). For the present study (Figures 1 and 2, Table 1, and Supplemental Table 1), patients enrolled in the WCM cohort up through 07/2016 (Figure 1) and a convenience sample of 199 patients enrolled in the BWH cohort between 07/2008 and 09/2013, for whom adequate aliquots of never-thawed plasma were available (Figure 2) were included.

Biological samples (including plasma) and extensive phenotypic data (such as demographics, medical history, laboratory parameters, and presence of sepsis) were prospectively collected (10-12). Collected clinical data were adjudicated by physicians (10-12). The recent Sepsis-3 definition was used for the diagnosis of sepsis (34).

Human plasma preparation and measurement. Within 48 hours after ICU admission, whole blood (10 ml) was drawn from each patient into EDTA-coated blood collection tubes (BD Pharmingen). At the time of blood draw, some patients were endotracheally intubated and received invasive MV; those patients comprised the "MV yes" group and the remaining patients comprised the "MV no" group shown in Figure 1, A, B, and D; Figure 2, A and C; and Supplemental Figure 7. In the "MV yes" group, blood samples were drawn within approximately 48 hours (with the exact timing not being known) after initiation of MV. The collected blood samples were kept at $4^{\circ} \mathrm{C}$ and centrifuged within 4 hours at $490 \mathrm{~g}$ for 10 minutes. Plasma was subsequently divided into aliquots and kept at $-80^{\circ} \mathrm{C}$. RIPK3 was assayed in duplicate using the Human Receptor-interacting serine/threonine-protein kinase 3 (RIPK3) ELISA kit (CSB-EL019737HU, CUSABIO) (18). Plasma was diluted 10 times, and the manufacturer's instructions were followed. RIPK1 was assayed in duplicate in 1:10 diluted plasma using the Human RIPK1/RIP ELISA Kit (Sandwich ELISA) (LS-F9254, LifeSpan BioSciences) in accordance with the manufacturer's instructions. Similarly, MLKL was assayed in duplicate in 1:10 diluted plasma using the Human MLKL ELISA Kit (Sandwich ELISA) (LS-F18428, LifeSpan BioSciences) in accordance with the manufacturer's instructions.

Mice. WT (C57BL/6N) mice were purchased from the Charles River Laboratories. Ripk3-/- mice were provided by Vishva Dixit and Kim Newton (Genentech). Generation of Ripk3-/- mice on a C57BL/6N background was described previously (35). Age- and sex-matched WT (C57BL/6N) mice were used as

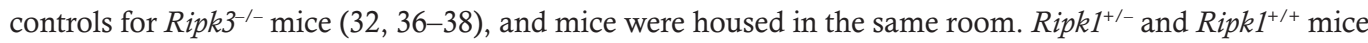
were provided by Michelle Kelliher (University of Massachusetts Medical School, Worcester, Massachusetts, USA) (20). $M l k t^{--}$and $M l k l^{+/+}$mice were provided by Jiahuai Han (School of Life Sciences, Xiamen

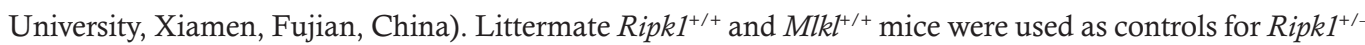
and $\mathrm{Mlkt}^{-1-}$ mice, respectively. Both male and female mice (8-15 weeks old) were used, unless otherwise specified. Sample sizes were determined based on our previous experience with VILI experiments (11). Experiments were not randomized and were not done in a blinded way. Animals were housed under identical pathogen-free conditions.

MV of mice and physiologic assessment of VILI. VILI, the most commonly used animal model of acute lung injury (39), was selected for these experiments, because of its high clinical relevance (1). Indeed, injurious MV in humans has been shown to result in a $9 \%$ absolute increase in mortality of critically ill patients with ARDS (2), while it has detrimental consequences even when applied in patients without ARDS (3-5). For induction of VILI, mice were anesthetized with an i.p. injection of pentobarbital $(70 \mathrm{mg} / \mathrm{kg})$. The trachea was exposed and a 19-gauge cannula was inserted and stabilized in the trachea lumen. A rodent computer-controlled piston ventilator (Flexivent, SCIREQ), which provides volume-controlled ventilation, was connected to the trachea cannula. According to a prespecified exclusion criterion, if peak airway pressure of the mouse at this starting point reached the machine's default peak pressure limit (i.e., $30 \mathrm{cmH}_{2} \mathrm{O}$ ), the mouse was not ventilated and was not considered for any analysis. Ventilated animals received either high tidal volume $(25 \mathrm{ml} / \mathrm{kg}$, injurious ventilation) or low tidal volume $(6 \mathrm{ml} / \mathrm{kg})$ of air through the ventilator. A positive end expiratory pressure of $2 \mathrm{cmH}_{2} \mathrm{O}$ was set. Mice were ventilated for 7 hours, unless otherwise specified. During the ventilation, a heat lamp was used to control the ambient temperature, and the core temperature of mice was measured using rectal thermometer. $0.2 \mathrm{ml} \mathrm{PBS} /$ animal/h of ventilation was administered i.p. to ensure adequate intravascular fluid volume. Likewise, supplemental doses of pentobarbital (one-third to one-half of the original dose/animal/h of ventilation) were administered i.p. to maintain deep anesthesia. Each hour of the ventilation, lungs were recruited by inflation with up to $30 \mathrm{cmH}_{2} \mathrm{O}$ pressure, and lung respiratory function parameters (including lung parenchymal tissue elastance, inspiratory capacity and tissue damping) were measured using the forced oscillation technique operated by FlexiWare 7 software. Changes in lung parenchymal tissue elastance, in inspiratory capacity, and in tissue damping were used for physiologic assessment of development of VILI. Change in lung elastance was defined as 
the lung elastance at the end of ventilation minus the average of lung elastance at 0 hours and at 1 hour. Change in inspiratory capacity was defined as the inspiratory capacity at the end of ventilation minus the average of inspiratory capacity at 0 hours and at 1 hour. Similarly, change in tissue damping was defined as the tissue damping at the end of ventilation minus the average of tissue damping at 0 hours and at 1 hour.

Retrieval of mouse BALF and measurements. After completion of MV, animals were euthanized by pentobarbital overdose (300 mg/kg i.p.). BAL was performed by instilling $1 \mathrm{ml}$ PBS into the trachea, and BALF (at least $0.7 \mathrm{ml}$ ) was retrieved with gentle suction. The lungs were perfused with PBS, inflated, and resected to be used for immunohistochemistry. BALF was centrifuged $(153 \mathrm{~g})$ for 5 minutes at $4^{\circ} \mathrm{C}$ to separate cellular components. The cell pellet was reconstituted in $1 \mathrm{ml}$ cold PBS, and total white cells were counted using a hemocytometer. BALF supernatant was used for measurement of total protein concentration (Pierce BCA Protein Assay Kit, 23225, Thermo Fisher Scientific), LDH (LDH-Cytotoxicity Colorimetric Assay Kit II, K313-500, BioVision), IL-6 (mouse IL-6 Quantikine ELISA Kit, M6000B, R\&D Systems), and HMGB1 (HMGB1 ELISA kit, ST51011, IBL International/TECAN) according to the manufacturer's instructions.

Treatment with necrostatin-1, perhexiline, and etomoxir. For the experiments involving necrostatin-1 (N9037, MilliporeSigma), necrostatin-1 [12.5 mg/kg mouse body weight, dissolved in dimethyl sulfoxide (DMSO) diluted in PBS] or vehicle (i.e., same volume of DMSO diluted in PBS) was administered i.p. 15 minutes before initiation of MV (40). For the experiments involving perhexiline (SML0120, MilliporeSig$\mathrm{ma}$ ), perhexiline maleate ( $8 \mathrm{mg} / \mathrm{kg}$ mouse body weight, dissolved in DMSO diluted in PBS) or vehicle (i.e., same volume of DMSO diluted in PBS) was administered i.p. 15 minutes before initiation of MV. The human equivalent of the dose that was administered in mice $(8 \mathrm{mg} / \mathrm{kg}$ mouse body weight is thought to be equivalent to $0.65 \mathrm{mg} / \mathrm{kg}$ human adult body weight) (41) is within the recommended daily dosage range of perhexiline for humans (50-400 mg) (42); in fact, it is close to the lower recommended dose for humans (42). We selected this low, yet clinically used, dose of perhexiline to reduce the possibility of overdose toxicity. Indeed, all mice receiving perhexiline and undergoing MV with low tidal volume survived for the whole duration of MV (Figure 7, A-C, and Supplemental Figure 8, A-C). For the experiments involving etomoxir (E1905, MilliporeSigma), etomoxir sodium salt hydrate (55 mg/kg mouse body weight, dissolved in PBS) or vehicle (i.e., same volume of PBS) was administered i.p. 30 minutes before initiation of MV (43). All mice receiving etomoxir and undergoing $\mathrm{MV}$ with low tidal volume survived for the whole duration of $\mathrm{MV}$ (Supplemental Figure 9, A-F).

Our pilot experiments had showed that, following treatment with CPT1 inhibitor (perhexiline or etomoxir), all mice undergoing MV with low tidal volume but not all mice undergoing MV with high tidal volume could survive for the whole duration of MV of 7 hours. In an attempt to address this issue, for the experiments involving perhexiline and etomoxir, we limited the duration of MV to 6 hours and 5 hours, respectively. As a result of the different duration of exposure to the injurious stimulus, the degree of VILI, as assessed by BALF protein, in control groups (WT mice without perhexiline or etomoxir exposed to MV with high tidal volume) of experiments involving CPT1 inhibitors (Figure 7B and Supplemental Figure 9D) was not as severe as the degree of VILI in control groups (WT mice exposed to MV with high tidal volume) in experiments not involving CPT1 inhibitor (Figure 3D). Despite the limited duration of MV to 6 hours, 4 of 8 mice receiving perhexiline and undergoing MV with high tidal volume (Figure 7B) did not survive for the whole duration of MV. However, BALF protein levels (and therefore degree of VILI) of the 4 mice that survived were comparable to those of the 4 mice that did not survive for the whole duration of MV (Figure 7B). All 8 above-mentioned mice (i.e., both those that survived and those that did not survive for the whole duration of MV) were analyzed and depicted in Figure 7B. Similarly and interestingly, all 11

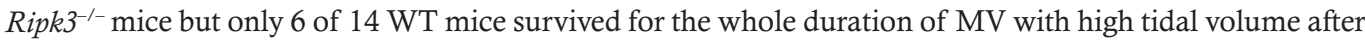
treatment with perhexiline. With the exception of one WT mouse, which had very high BALF protein $(>4$ times higher than median) after treatment with perhexiline and exposure to MV with high tidal volume, all above-mentioned mice were analyzed and depicted in Figure 7E.

Immunohistochemistry. Monoclonal rabbit anti-RIPK3 antibody for mouse RIPK3 (15828, Cell Signaling) at a 1:100 dilution was used for staining of formalin-fixed, paraffin-embedded lung tissue sections (Supplemental Figure 2).

Metabolomic analysis in humans. Metabolomic profiles had been previously generated from plasma samples of 90 ICU patients on day 1 of their enrollment in the BWH Registry of Critical Illness (24). Additional details on the 90 patients and the metabolomic analysis by Metabolon Inc. (using gas and liquid chromatography and mass spectroscopy) have been published (24). For the present study (Figure 5 and 
Supplemental Figure 7), a secondary analysis of raw data on 3 prespecified metabolites (namely, free carnitine, palmitoylcarnitine, and oleylcarnitine) was carried out for patients with versus without ARDS (Figure $5 \mathrm{~A}$ ) and for patients with versus without MV (Supplemental Figure 7). After normalization to median, the $\mathrm{C} 0 /(\mathrm{C} 16+\mathrm{C} 18)$ ratio was calculated for 86 patients (Figure 5A and Supplemental Figure 7). Four patients were excluded because they had a high plasma creatinine level $(>5 \mathrm{~g} / \mathrm{dl})$, which affects the carnitine levels (44). We had available samples to measure plasma RIPK3 levels in 39 patients of the above-mentioned 90 patients (24) for whom metabolomic data were available; these 39 patients were included in the Figure 2 . In other words, for 39 of the 199 patients enrolled in the BWH cohort (Figure 2), we had both metabolomic and RIPK3 data in plasma. The median RIPK3 level of these 39 patients was $2,012 \mathrm{pg} / \mathrm{ml}$. One patient was excluded because he/she had a high plasma creatinine level ( $>5 \mathrm{~g} / \mathrm{dl}$ ), which affects the carnitine levels (44). We categorized the remaining 38 patients into two groups; one group of patients had plasma RIPK3 levels equal to or lower than median ("low RIPK3" group), and the other group of patients had plasma RIPK3 levels higher than median ("high RIPK3" group) (Figure 5B). We found that the C0/(C16+C18) ratio was higher in the plasma of patients in the "high RIPK3" group compared with the "low RIPK3" group (Figure $5 \mathrm{~B}$ ), suggesting an association between the increase in plasma RIPK3 levels and the increase in the $\mathrm{C} 0 /(\mathrm{C} 16+\mathrm{C} 18)$ ratio in critically ill patients. Increase in the $\mathrm{C} 0 /(\mathrm{C} 16+\mathrm{C} 18)$ ratio is thought to reflect impaired FAO (45) and is clinically used as newborn screening test for CPT1 deficiency (25).

Metabolomic analysis in mice. WT mice (C57BL/6, male, 8-12 weeks old) underwent injurious (tidal volume of $12 \mathrm{ml} / \mathrm{kg}$ ) or no MV ( $n=8 /$ group). Lungs were perfused with PBS, harvested, and immediately stored at $-80^{\circ} \mathrm{C}$. Metabolomic profiling of the collected lung specimens (Figure 6A) was carried out by Metabolon Inc., which uses gas chromatography and liquid chromatography combined with detection by mass spectroscopy, as described previously (46).

Fatty acid assay. The Free Fatty Acid Quantification Colorimetric/Fluorometric Kit (K612-100, Biovision) was used to measure free fatty acid levels in BALF of mice undergoing MV with low or high tidal volume (Figure 6, B and C). Fifty $\mu 1$ of undiluted BALF, collected as described above, was used, and the manufacturer's instructions were followed.

Statistics. Continuous variables were presented as mean \pm SEM and compared with the nonparametric Mann Whitney $U$ test (for comparison of two groups) or the ANOVA with Tukey post hoc correction (for comparisons of more than two groups). Categorical variables were presented as percentages and compared with the Fisher's exact or $\chi^{2}$ test. Pearson's correlation analysis was used in Figure 1, C and E, and in Figure 6C. All analyses, except the multiple linear regression analysis displayed in Supplemental Table 1, were carried out using GraphPad Prism 5.01 (GraphPad Software Inc.). The multiple linear regression analysis was carried out using STATA 14.2 (StataCorp LP) to isolate the contribution of sex, age, malignancy, MV, vasopressors, and sepsis (independent variables) to the plasma levels of RIPK3 (continuous dependent variable). A 2-tailed $P$ of less than 0.05 was considered to denote statistical significance.

Study approval. The registries of critically ill patients were approved by Institutional Review Board of WCM (1405015116A005) and by the Partners Human Research Committee (2008-P-000495). Both registries enrolled patients who provided written informed consent for blood collection. All mouse experimental protocols were approved by the Institutional Animal Care and Use Committee of Weill Cornell Medical College (protocol 2013-0108).

\section{Author contributions}

IIS and AMKC conceived the study with assistance from KN; IIS, KCM, RMB, LEF, EJF, MTL, and EJS contributed the experiments for human subjects; IIS, MI, JWH, JSM, DSJ, and SWR contributed the in vivo experiments; IIS and AMKC wrote the paper; and AMKC supervised the entire project.

\section{Acknowledgments}

The authors would like to acknowledge the following members of the BWH Registry of Critical Illness: Paul Dieffenbach, Samuel Ash, Joshua Englert, Diana Barragan Bradford, Carolina Quintana, and Angelica Higuera. The authors also acknowledge Clara Oromendia (WCM) for statistical advices. Finally, the authors acknowledge Michelle Kelliher (University of Massachusetts Medical School) for providing Ripk1 $1^{+/-}$and Ripk1 ${ }^{+/+}$mice and Jiahuai Han (School of Life Sciences, Xiamen University) for providing $\mathrm{Mlkl}^{-/-}$and $\mathrm{Mlkl}^{+/+}$mice. This work was supported by the US National Institutes of Health (grant P01 HL108801, R01 HL079904, R01 HL055330, all to AMKC). 
Address correspondence to: Augustine M.K. Choi, Weill Cornell Medicine, 525 East 68th Street, Room F-104, New York, New York 10065, USA. Phone: 212.746.6005; Email: amc2056@med.cornell.edu.

1. Slutsky AS, Ranieri VM. Ventilator-induced lung injury. N Engl J Med. 2013;369(22):2126-2136.

2. Acute Respiratory Distress Syndrome Network, et al. Ventilation with lower tidal volumes as compared with traditional tidal volumes for acute lung injury and the acute respiratory distress syndrome. N Engl J Med. 2000;342(18):1301-1308.

3. Futier E, et al. A trial of intraoperative low-tidal-volume ventilation in abdominal surgery. $N$ Engl J Med. 2013;369(5):428-437

4. Serpa Neto A, et al. Association between use of lung-protective ventilation with lower tidal volumes and clinical outcomes among patients without acute respiratory distress syndrome: a meta-analysis. JAMA. 2012;308(16):1651-1659.

5. Guay J, Ochroch EA. Intraoperative use of low volume ventilation to decrease postoperative mortality, mechanical ventilation, lengths of stay and lung injury in patients without acute lung injury. Cochrane Database Syst Rev. 2015;12(12):CD011151.

6. Imai $Y$, et al. Injurious mechanical ventilation and end-organ epithelial cell apoptosis and organ dysfunction in an experimental model of acute respiratory distress syndrome. JAMA. 2003;289(16):2104-2112.

7. Hammerschmidt S, Kuhn H, Grasenack T, Gessner C, Wirtz H. Apoptosis and necrosis induced by cyclic mechanical stretching in alveolar type II cells. Am J Respir Cell Mol Biol. 2004;30(3):396-402.

8. Pasparakis M, Vandenabeele P. Necroptosis and its role in inflammation. Nature. 2015;517(7534):311-320.

9. Weinlich R, Oberst A, Beere HM, Green DR. Necroptosis in development, inflammation and disease. Nat Rev Mol Cell Biol. 2017;18(2):127-136

10. Finkelsztein EJ, et al. Comparison of qSOFA and SIRS for predicting adverse outcomes of patients with suspicion of sepsis outside the intensive care unit. Crit Care. 2017;21(1):73.

11. Dolinay T, et al. Inflammasome-regulated cytokines are critical mediators of acute lung injury. Am J Respir Crit Care Med. 2012;185(11):1225-1234.

12. Nakahira K, et al. Circulating mitochondrial DNA in patients in the ICU as a marker of mortality: derivation and validation. PLoS Med. 2013;10(12):e1001577.

13. Moreno-Gonzalez G, Vandenabeele P, Krysko DV. Necroptosis: A novel cell death modality and its potential relevance for critical care medicine. Am J Respir Crit Care Med. 2016;194(4):415-428

14. Newton K, et al. RIPK3 deficiency or catalytically inactive RIPK1 provides greater benefit than MLKL deficiency in mouse models of inflammation and tissue injury. Cell Death Differ. 2016;23(9):1565-1576.

15. Siempos II, et al. Pretreatment with atorvastatin attenuates lung injury caused by high-stretch mechanical ventilation in an isolated rabbit lung model. Crit Care Med. 2010;38(5):1321-1328.

16. Zhang T, et al. CaMKII is a RIP3 substrate mediating ischemia- and oxidative stress-induced myocardial necroptosis. Nat Med. 2016;22(2):175-182.

17. Mizumura K, et al. Mitophagy-dependent necroptosis contributes to the pathogenesis of COPD. J Clin Invest. 2014;124(9):3987-4003

18. Qing DY, et al. Red blood cells induce necroptosis of lung endothelial cells and increase susceptibility to lung inflammation. $A m$ J Respir Crit Care Med. 2014;190(11):1243-1254.

19. Cho YS, et al. Phosphorylation-driven assembly of the RIP1-RIP3 complex regulates programmed necrosis and virus-induced inflammation. Cell. 2009;137(6):1112-1123.

20. Kelliher MA, Grimm S, Ishida Y, Kuo F, Stanger BZ, Leder P. The death domain kinase RIP mediates the TNF-induced NF-kappaB signal. Immunity. 1998;8(3):297-303.

21. Degterev A, et al. Identification of RIP1 kinase as a specific cellular target of necrostatins. Nat Chem Biol. 2008;4(5):313-321.

22. Sun L, et al. Mixed lineage kinase domain-like protein mediates necrosis signaling downstream of RIP3 kinase. Cell. 2012;148(1-2):213-227.

23. Magtanong L, Ko PJ, Dixon SJ. Emerging roles for lipids in non-apoptotic cell death. Cell Death Differ. 2016;23(7):1099-1109.

24. Rogers AJ, et al. Metabolomic derangements are associated with mortality in critically ill adult patients. PLoS One. 2014;9(1):e87538.

25. Newborn Screening ACT Sheet: [Elevated C0/C16+C18], Carnitine Palmitoyl Transferase 1 Deficiency (CPT1). American College of Medical Genetics. https://www.acmg.net/StaticContent/ACT/C0_C16-C18.pdf. Assessed April 9, 2018.

26. Carracedo A, Cantley LC, Pandolfi PP. Cancer metabolism: fatty acid oxidation in the limelight. Nat Rev Cancer. 2013;13(4):227-232

27. Kang HM, et al. Defective fatty acid oxidation in renal tubular epithelial cells has a key role in kidney fibrosis development. Nat Med. 2015;21(1):37-46.

28. Phan TT, et al. Multi-centre experience on the use of perhexiline in chronic heart failure and refractory angina: old drug, new hope. Eur J Heart Fail. 2009;11(9):881-886.

29. Lee L, et al. Metabolic modulation with perhexiline in chronic heart failure: a randomized, controlled trial of short-term use of a novel treatment. Circulation. 2005;112(21):3280-3288.

30. Holubarsch CJ, et al. A double-blind randomized multicentre clinical trial to evaluate the efficacy and safety of two doses of etomoxir in comparison with placebo in patients with moderate congestive heart failure: the ERGO (etomoxir for the recovery of glucose oxidation) study. Clin Sci. 2007;113(4):205-212.

31. Kearney CJ, Martin SJ. An inflammatory perspective on necroptosis. Mol Cell. 2017;65(6):965-973.

32. Imamura M, et al. RIPK3 promotes kidney fibrosis via AKT-dependent ATP citrate lyase. JCI Insight. 2018;3(3):e94979.

33. Khan MJ, et al. Inhibition of autophagy rescues palmitic acid-induced necroptosis of endothelial cells. J Biol Chem. 2012;287(25):21110-21120.

34. Singer M, et al. The third international consensus definitions for sepsis and septic shock (Sepsis-3). JAMA. 2016;315(8):801-810

35. Newton K, Sun X, Dixit VM. Kinase RIP3 is dispensable for normal NF-kappa Bs, signaling by the B-cell and T-cell receptors, tumor necrosis factor receptor 1, and Toll-like receptors 2 and 4. Mol Cell Biol. 2004;24(4):1464-1469. 
36. Seifert L, et al. The necrosome promotes pancreatic oncogenesis via CXCL1 and Mincle-induced immune suppression. Nature. 2016;532(7598):245-249.

37. Günther C, et al. The pseudokinase MLKL mediates programmed hepatocellular necrosis independently of RIPK3 during hepatitis. J Clin Invest. 2016;126(11):4346-4360.

38. Dara L, et al. Receptor interacting protein kinase 1 mediates murine acetaminophen toxicity independent of the necrosome and not through necroptosis. Hepatology. 2015;62(6):1847-1857.

39. Matute-Bello G, Frevert CW, Martin TR. Animal models of acute lung injury. Am J Physiol Lung Cell Mol Physiol. 2008;295(3):L379-L399.

40. Takemoto K, et al. Necrostatin-1 protects against reactive oxygen species (ROS)-induced hepatotoxicity in acetaminophen-induced acute liver failure. FEBS Open Bio. 2014;4:777-787.

41. Reagan-Shaw S, Nihal M, Ahmad N. Dose translation from animal to human studies revisited. FASEB J. 2008;22(3):659-661.

42. Horowitz JD, Sia ST, Macdonald PS, Goble AJ, Louis WJ. Perhexiline maleate treatment for severe angina pectoris--correlations with pharmacokinetics. Int J Cardiol. 1986;13(2):219-229.

43. Samudio I, et al. Pharmacologic inhibition of fatty acid oxidation sensitizes human leukemia cells to apoptosis induction. J Clin Invest. 2010;120(1):142-156.

44. Parikh S, et al. A modern approach to the treatment of mitochondrial disease. Curr Treat Options Neurol. 2009;11(6):414-430.

45. DiMauro S, Hirano M, Schon EA. Approaches to the treatment of mitochondrial diseases. Muscle Nerve. 2006;34(3):265-283.

46. Evans AM, DeHaven CD, Barrett T, Mitchell M, Milgram E. Integrated, nontargeted ultrahigh performance liquid chromatography/electrospray ionization tandem mass spectrometry platform for the identification and relative quantification of the small-molecule complement of biological systems. Anal Chem. 2009;81(16):6656-6667. 